\title{
A Fast Channel Switching Scheme for IPTV Live Broadcasting Service
}

\author{
Zhang Yibo \\ Chongqing College of Electronic Engineering, Chongqing, 401331, China \\ Emal: 534878774@qq.com
}

Keywords: IPTV; Channel Switching; Server; Multicast.

\begin{abstract}
With the development of information technology, the application of network TV service has been developed rapidly. Reducing the channel switching time is the key factor to improve the quality of service, and is also one of the problems to be solved in the large-scale application of IPTV service, on the basis of summarizing the main channel switching schemes at present, an improved fast channel switching scheme is proposed in this paper. A temporary channel is used to speed up the transmission of streaming media in the process of media transmission, and a double buffer is used to store the media data in the transmission terminal to realize the fast switching between different channels.
\end{abstract}

\section{Introduction}

With the progress of network technology, the application of IPTV business has been gradually promoted. Direct broadcast service is the basic business of IPTV, and channel switch delay is one of the important indicators of its service quality. Channel switching time refers to the time required from the user to submit a channel switching request to the screen of the requested channel to be displayed to the user on the television screen. At present, the channel switching time of cable TV is about 0.5 seconds, and the switching time of digital TV is slightly longer than that of cable TV, about 1 second. The research results show that in IPTV services, the upper limit of channel switching time that users can accept is 2 seconds, and users who exceed 2 seconds will have a clear sense of lag. Due to the limitation of network bandwidth, IPTV's live channel service adopts multicast mode. Channel source only sends the video source which is currently playing to the user. When the user wants to switch the channel, he has to go through a series of request processing and data transmission to switch to the next channel. In this way, the channel switching delay of IPTV is difficult to meet the demand of 2 seconds.

\section{Analysis of mechanism in IPTV channel switching}

For IPTV live broadcast system, channel switching time is usually composed of the following parts:

(1) Channel information acquisition time: the change time can be omitted by downloading the EPG (Electronic Program Guide) file corresponding to the channel in advance;

(2) Time to get the first information frame (T1): In engineering, the interval of encoder I frame is generally set to 2-3 seconds, so the average time to get the first I frame is 1-1.5 seconds.

(3) Re-initialization time of the terminal encoder: it can be ignored because it is generally in tens of milliseconds and can be carried out simultaneously with the receiving I frame.

(4) Terminal buffer delay playback time (T2): usually 1-2 seconds.

It can be seen that T1 and T2 are the main factors affecting the channel switching time, and the improvement of channel switching is mainly in these two aspects. At present, the following two main categories are included:

\subsection{Fast switching scheme based on video typical frame extraction}

The basic idea of this scheme is to provide users with a short typical frame sequence of a new channel quickly when they switch channels, so as to improve users' sense of delay in IPTV channel 
switching. It is necessary to add a live server to the network side of the system, which receives and processes the data of all live channels in real time. When the user switches the channel, the terminal sends a request to the live broadcast processing server while quitting the normal multicast. The live broadcast server is correspondingly and quickly provided to the user with a short sequence of typical frames of the new channel. After the typical frame sequence reaches the terminal buffer, it can be decoded and output. When the packet of the new normal channel reaches the terminal buffer, the terminal decodes and outputs the data of the new channel. If the typical frame is the video frame before the current time of the new channel, or close to the current time of the video frame, then the user experience will feel that the switch is smooth, improve user perception.

In this scheme, a typical frame is sent to the terminal by the live processing server before the normal packet arrives at the terminal. This typical frame can not guarantee the continuity of the packets with the normal new channel, and the picture will be discontinuous at the terminal; moreover, a live processing server is added to increase the complexity of the network.

\section{2 channel switching based on retransmission channel}

This scheme establishes and maintains a persistent TCP retransmission channel between terminal and streaming media server. The terminal first obtains the nearest or next I frame and subsequent frame sequence from the streaming media server's cache through the retransmission channel at a bit rate greater than the decoding speed, so that the resulting stream will lag at least one I frame behind the multicast stream, and the terminal will output the video immediately after receiving the first I frame without waiting for the cache. At this point, because the transmission speed is faster than the decoding speed, the decoding buffer of the terminal will be gradually filled up, and the cache of the streaming media server will be consumed gradually. Therefore, when the unicast stream transmits the latest bit stream data (at this time the unicast stream is ahead of the multicast stream), it is necessary to instruct the terminal to switch to the receiving multicast stream. Since the multicast data that started receiving was duplicated with the original unicast data, it was discarded until the new serial number data was received, thus achieving a smooth convergence.

This scheme requires a streaming media server to notify the terminal of multicast stream switching. It is uncertain whether the multicast stream and unicast stream have overlapped the server, and increases the burden of the streaming media server.

\section{Fast channel switching scheme}

The improvements described above mainly optimize the server on the network side, and the specific playback situation on the user terminal is unknown to the server, so that the IPTV terminal side is always in a passive position. IPTV terminals (including set-top boxes) are intelligent terminals with high performance. This paper focuses on the terminal side of the proposed terminal and media server to cooperate with the fast channel switching scheme, the basic structure as shown in Figure 1.

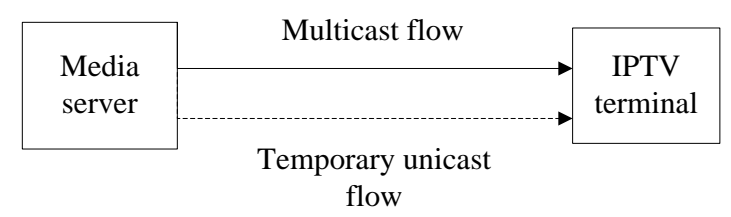

Fig. 1 Structure diagram

The basic idea of the scheme proposed in this paper is that each time the channel is switched, a normal multicast channel is established between the server and the terminal for transmitting normal live media streams, and a temporary unicast channel is also established. This channel is temporary for fast establishment of the playback process. When the temporary channel is released is notified to the server by the terminal based on media reception, and how the terminal determines it will be explained in the next section. The unicast channel sends the frame sequence in the streaming media server cache to the terminal as quickly as possible. If the buffer is empty and no release request 
from the terminal is received, the frame sequence is sent at the normal speed. The specific process of channel switching is shown in Figure 2.

Firstly, the media information of the new channel is obtained, which can be parsed from EPG (Electric Program Guide) in advance. The information contains the multicast address of the new channel and a unicast address. When the user requests channel switching, the terminal first exits the original multicast group, then obtains the multicast address of the new channel from the local data, and joins the new multicast group to the broadband access device through IGMP to receive the live media stream. At the same time, the terminal sends the session request to the server to establish the unicast channel. After the session is established, the terminals begin to receive live broadcast and unicast media streaming simultaneously. The terminal establishes two independent buffers for multicast and unicast streams respectively. The data in the two buffers which can display faster will be decoded and displayed to the user. At the same time, comparing the two streams of packets, when it is detected that the data in the two buffers has reached a complete overlap, it is determined that unicast streams can be disconnected, a request can be sent to the server, temporary unicast channel can be released, unicast streams can be deleted. At this point, the channel switching process ends and enters the normal live broadcast stage.

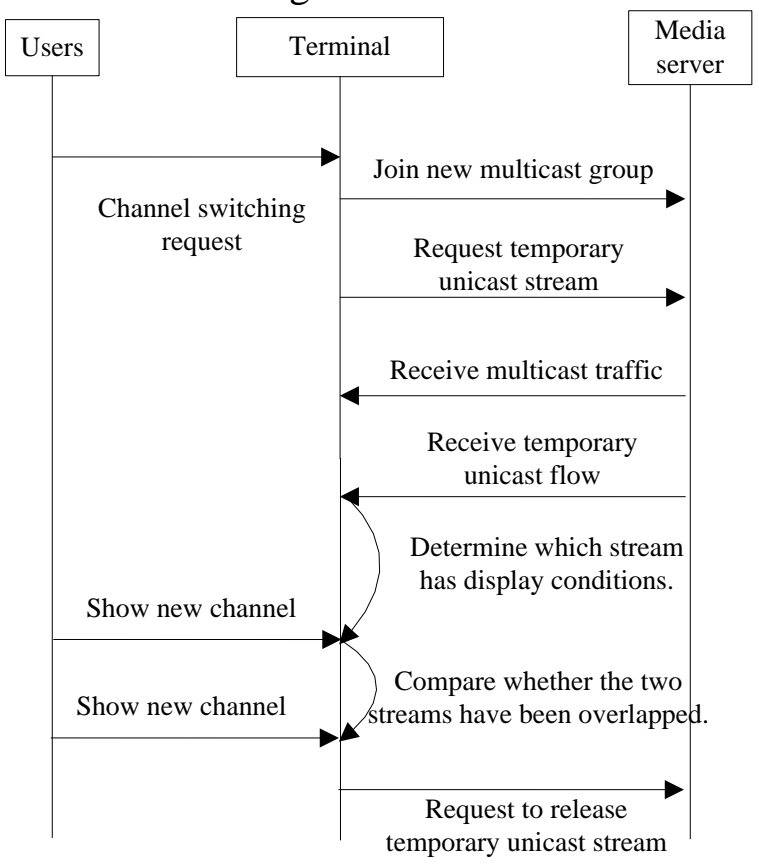

Fig.2 Flow chart of channel switching

In order to alleviate the delay and jitter of network, we need to introduce buffer into IPTV terminal. In the playback process of the terminal, the data stream sent by the server reaches the buffer of the playback terminal through the network, and then sends it to the decoding and playback module for decoding and displaying. Usually, a receiving thread is started to receive the video data, and a decoding and playback thread is started to decode and playback the video data. In order to implement the fast handoff scheme proposed in this paper, we set up two separate buffers for multicast and unicast streams, and receive two media streams simultaneously. A comparison thread needs to be started to compare the two media. The task of this thread is to determine which stream has the display condition first and which buffer data is sent to the decoding module. The judgment is based on which stream receives the I frame first, because I frame adopts intra-town coding method, that is, only uses the spatial correlation in a single image, without using the temporal correlation, decoding without reference to other frames can be independent decoding display. Although theoretically temporary unicast streams will be the first to have display conditions, it is necessary to make a judgment, because two streams pass through different IP routes, due to different network conditions, unicast streams can not be guaranteed to arrive first. Then the thread compares whether the data in the two buffers overlaps, because MPEG2 is the current standard for 
HDTV in China. Media data is sent using TS (Transport Stream) streams and can be achieved by comparing the DTS (decoding time-stamp) of each frame of data. The comparison flowchart is shown in Figure 3.

When the two buffers' data overlap, a request is sent to the server to release the temporary unicast stream to send the multicast stream data to the decoder, and the comparison thread ends.

In the overall structure, in order to make the terminal receive the media stream of the new channel at the fastest speed, a temporary unicast channel is added at the beginning of the switch. Through this unicast channel, the server sends the media data directly to the terminal, which can meet the requirements of fast display. The unicast channel is temporary. Although the traffic on the terminal network increases briefly within 2 seconds of the channel switching, the unicast channel is released and the data flow is quickly restored to normal state after the switching is completed.

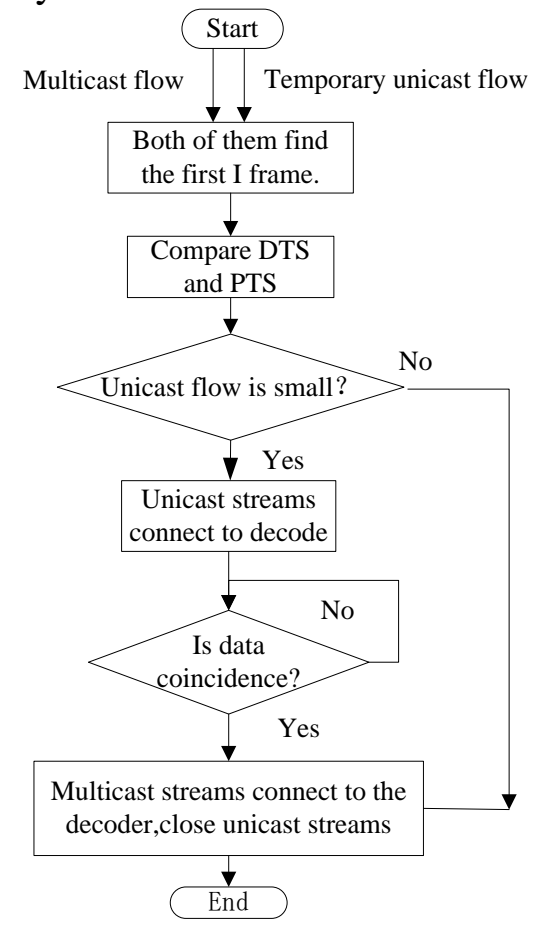

Fig. 3 Flow chart of buffer data comparison

From the point of view of release time of temporary unicast channel, the terminal always compares two media streams during channel switching. Once it finds that the media streams overlap, it notifies the server and requests unicast streams to be released. The server receives the release request from the terminal, which shows that the two streams on the terminal do overlap, and also improves the role of the terminal in the channel switching process.

The proposed scheme is tested by VLS software. Normal unicast stream is used instead of temporary unicast stream. Because the data frame is not pure I frame, the measured channel switching time will be longer than the actual switching time of the scheme. The average channel switching time of 50 experiments is 1.187s. Except for very few cases, the switching time fluctuates greatly. In most cases, the switching time is between 1.0 and $1.4 \mathrm{~s}$, which meets the upper limit of IPTV channel switching time.

\section{Conclusion}

In this paper, a channel switching scheme based on IPTV terminal and streaming media server is proposed. This scheme utilizes the high performance of IPTV terminal to realize some functions of channel switching by the terminal, which improves the speed of channel switching and reduces the burden of server. At the same time, the temporary unicast channel also reduces the network traffic. The scheme needs terminal coordination, which has certain requirements for terminal performance. 


\section{References}

[1] MA Shaolin, LI Wenyao. IPTV Fast Channel Change Technology Based on Server[J].TV Engineering, 2012 , 36 (24) :57-60.

[2] Weng Yi, Yao Liang, Xi Xi. Research on a Unicast-Based IPTV Channel Change Solution [J].Telecommunications Science, 2014, 30 (9):159-163.

[3] GUO Jun, WANG Yu. On Instant Channel Changing Solution for IP TV and Its Realization [J]. Tianjin Science \& Technology, 2014, 41 (6):29-32.

[4] ZHANG Peng, YANG Qian-bin, ZHANG Xing-ming, et al.Design of Channel Zapping Supporting IPTV Subscriber Increasing [J]. Computer Engineering, 2009, 35(3):99-102. 